\title{
Effect of halometasone cream combined with Simiao pill on the therapeutic effect and expression of serum leukotriene B4 (LTB4) and thymic stromal lymphopoietin (TSLP) in patients with eczema, and the factors influencing its clinical efficacy
}

\author{
Jun Cao ${ }^{1}$, Hui Liu ${ }^{1}$, Xiao-Jie Ding ${ }^{2}$ \\ ${ }^{1}$ Department of Dermatology, Wuxi 9th Affiliated Hospital of Soochow University, Wuxi, China; ${ }^{2}$ Department of Dermatology, The Second \\ Affiliated Hospital of Nanjing University of Chinese Medicine, Jiangsu Second Chinese Medicine Hospital, Nanjing, China \\ Contributions: (I) Conception and design: J Cao; (II) Administrative support: XJ Ding; (III) Provision of study materials or patients: H Liu; (IV) \\ Collection and assembly of data: J Cao; (V) Data analysis and interpretation: All authors; (VI) Manuscript writing: All authors; (VII) Final approval of \\ manuscript: All authors. \\ Correspondence to: Xiao-Jie Ding. Department of Dermatology, the Second Affiliated Hospital of Nanjing University of Chinese Medicine, No. 336, \\ Shuiximen street, Jianye District, Nanjing 210000, China. Email: xiaonail_123@163.com.
}

Background Chronic eczema has the characteristics of a long treatment cycle and repeated attacks, which
seriously affects the daily life and work of patients. Topical glucocorticoids are the first-line treatment for
chronic eczema. This study aimed to retrospectively analyze the effects of halometasone cream combined
with Simiao pill on the efficacy and expression of serum leukotriene B4 (LTB4) and thymic stromal
lymphopoietin (TSLP) in patients with eczema, and identify the factors influencing its clinical efficacy.
Methods: We retrospectively collected the medical records of 195 patients with chronic eczema treated in
the dermatology department from January 2020 to May 2021 , and divided them into two groups according
to medication: 98 cases were treated with halometasone cream (control group) and 97 cases were treated with
halometasone ointment combined with Simiao pill (observation group). The severity of eczema, quality of
life, clinical efficacy, LTB4 and TSLP levels, and safety were compared between the two groups. Multivariate
logistic regression analysis was used to determine the independent factors affecting clinical efficacy.

Results: After treatment, the Eczema area and severity index (EASI) and Dermatology Life Quality Index (DLQI) scores in the observation group were markedly lower than those of the control group $(\mathrm{P}<0.05)$. The total clinical effective rate of the observation group was $88.8 \%$, which was notably higher than that of the control group 70.1\% ( $\mathrm{P}=0.001)$. The concentrations of serum LTB4 and TSLP in the observation group were markedly lower than those in the control group $(\mathrm{P}<0.05)$. Logistic regression analysis showed that the treatment regimen, digestive system symptoms, heavy aching limbs, and damp-heat tongue and pulse were independent factors affecting the curative effect of the patients $(\mathrm{P}<0.05)$.

Conclusions: Simiao pill combined with halometasone cream can effectively improve chronic eczema and enhance the clinical efficacy of treatment, which may be related to the reduction of serum LTB4 and TSLP levels. The treatment plan, digestive system symptoms, heavy aching limbs, and damp-heat tongue and pulse are the main factors that affecting the clinical curative effect. Thus, clinical intervention programs should be made according to the above factors to improve the quality of life of patients.

Keywords: Chronic eczema; halometasone cream; Simiao pill; clinical efficacy; influencing factor

Submitted Aug 31, 2021. Accepted for publication Oct 29, 2021.

doi: 10.21037/apm-21-2685

View this article at: https://dx.doi.org/10.21037/apm-21-2685

(c) Annals of Palliative Medicine. All rights reserved. 


\section{Introduction}

Chronic eczema is a common clinical skin disease, which is mainly induced by the comprehensive effects of food, infection source, endocrine changes, and genetic factors. It has the characteristics of a long treatment cycle and repeated attacks, and manifests clinically as pruritus, thickened, and lichen-like skin lesions, which seriously affect the daily life and work of patients $(1,2)$. Topical glucocorticoids are the first-line therapy for chronic eczema, among which halometasone cream is the most common. As a trihalonic acid compound, it can specifically combine with a steroid structure and play a role in changing protein synthesis and inhibiting the inflammatory response. It has been widely used in the treatment of chronic eczema diseases, yet its treatment cycle has not been accurately understood. Treatment that is applied for too long can easily cause local skin atrophy, while a treatment period that is too short can easily result in relapse $(3,4)$. It is suggested to avoid the use of high-dose Halometasone Cream in the treatment of chronic systemic eczema and use local lowdose Halometasone Cream to alleviate the symptoms of chronic systemic eczema (5).

Traditional Chinese medicine research $(6,7)$ has shown that the etiology of chronic eczema is spleen and stomach injury, abnormal damp heat, and intolerable endowment. Simiao pill contains traditional Chinese medicine such as Rhizoma Smilacis Glabrae, Cortex Phellodendri, Radix Salviae Miltiorrhizae, Cortex Eucommiae, Radix Achyranthis Bidentatae and Radix Glycyrrhizae, has the effects of dredging muscles and veins, clearing heat, and drying dampness, and can symptomatically treat chronic skin diseases. However, there are relatively few studies on the clinical effect and mechanism of action of Simiao pill combined with halometasone cream. Therefore, this paper aims to investigate the clinical efficacy and influencing factors of halometasone cream combined with Simiao pill, in order to provide a basis for clinical treatment and prevention of chronic eczema. We present the following article in accordance with the STROBE reporting checklist (available at https://dx.doi.org/10.21037/apm-21-2685).

\section{Methods}

\section{Patient selection}

The study was approved by the Ethics Committee of Wuxi 9th Affiliated Hospital of Soochow University (2020131) and was conducted in accordance with the Declaration of Helsinki (as revised in 2013). All participants provided informed consent. We retrospectively collected the medical records of 195 patients with chronic eczema treated in the dermatology department of Wuxi 9th Affiliated Hospital of Soochow University from January 2020 to May 2021, and divided them into two groups according to medication: 98 cases were treated with halometasone cream (control group) and 97 cases were treated with halometasone ointment combined with Simiao pill (observation group) (Figure 1).

The inclusion criteria were as follows: (I) patients diagnosed with chronic eczema according to the "Guide of Eczema Media" (8), and confirmed by pathological examination; (II) patients with clinical manifestations of the disorder, such as papules, plaques, and desquamation; (III) patients who did not take hormones, immunosuppressants, and other drugs within 1 month of treatment; and (IV) patients with complete case data. The exclusion criteria were as follows: (I) patients complicated with coagulation dysfunction, hemorrhagic disease, or autoimmune disease; (II) patients with liver, kidney, heart, and other important organ failure; (III) patients with other skin diseases; (IV) those who are allergic or intolerant to Halometasone Cream or Simiao pill; and (V) those with incomplete case data.

\section{Therapeutic method}

The patients included in the study were treated by the same nursing team. The patients in the control group were externally applied with halometasone cream (Aomei, China, approval number: hc20150050, batch number: 6201722). The medication was applied twice a day at $12 \mathrm{~h}$ intervals. The dosage refers to the principle of interphalangeal unit; the dosage of the joint length from the end of the index finger to the distal end is approximately $0.5 \mathrm{~g}$. This was applied evenly once on both palms, according to $1 \%$ body surface area as 1 palm area, and the dosage of the patient were calculated. Based on the patients in the control group, the patients in the observation group took Simiao pills (Jilin Zixin Pharmaceutical Co., Ltd., China, approval No. gyzzz22024960, batch No. 201101), twice a day, $6 \mathrm{~g}$ each time. Both groups were treated for 1 month.

\section{Observation index}

\section{Eczema lesion severity}

The Eczema area and severity index (EASI) (9) was used to evaluate the severity of lesions in the two groups before and after treatment. The specific morphology of the lesions was 


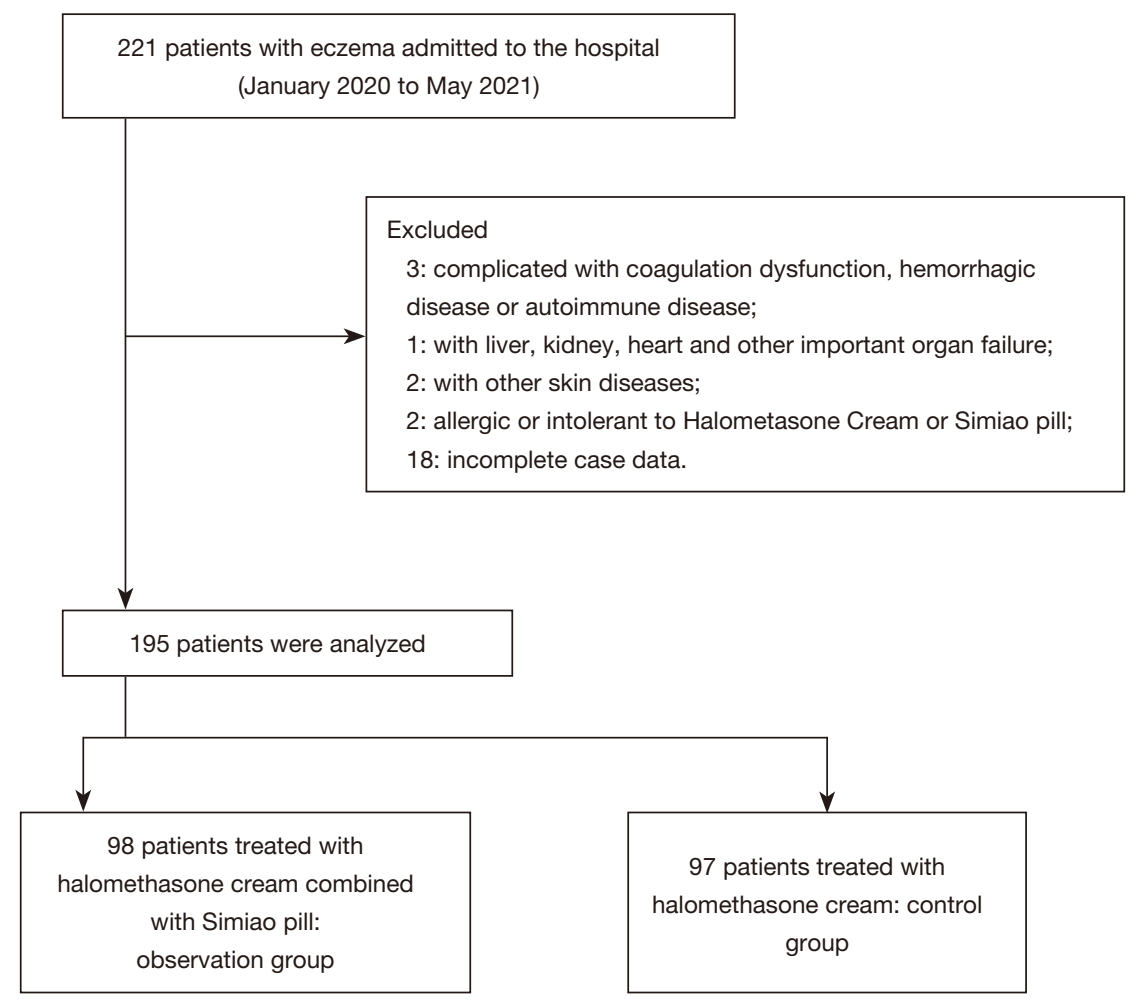

Figure 1 Patient selection flowchart.

as follows: clinical manifestations such as erythema, moss, scleredema, and epidermal exfoliation. This was scored according to the following method: 0 point for no lesions, 1 point for unable to confirm lesions after careful observation, 2 points for confirmed lesions after careful observation, and 3 points for immediately confirmed lesions. The skin lesion area score was divided into head and neck, upper limbs, lower limbs, and trunk; no skin lesion was scored 0 , less than $10 \%$ was scored $1,10-19 \%$ was scored $2,20-49 \%$ was scored 3, 50-69\% was scored 4, 70-89\% was scored 5, and $90-100 \%$ was scored 6 . The higher the score, the more severe the form of skin lesion, and the larger the area of skin lesion.

\section{Quality of life}

The Dermatology Life Quality Index (DLQI) (10) was used to evaluate the quality of life of the two groups before and after treatment. The scale included family, physiology, psychology, treatment, social activities, and interpersonal communication. There were 10 items in total, each with a score of $0-3$. The lower the score, the better the quality of life of the patients. The quality of life scale was distributed to patients before the first treatment and one month after treatment.

\section{Clinical efficacy}

In this paper, clinical efficacy (11) was evaluated by the efficacy index composed of the pruritus and EASI scores. The comprehensive score of pruritus was evaluated by degree, location and duration of pruritus. Each of these dimensions is scored between 1 and 3 points, and the scores between degrees can be expressed by 0.5 points. Curative effect index $=$ score difference before and after treatment / score before treatment $\times 100 \%$; Cure: the skin lesion morphology and clinical symptoms completely disappeared, and the curative effect index exceeded $95 \%$; Remarkable effect: the shape of skin lesions basically subsided, the clinical symptoms were significantly improved, and the curative effect index ranged from 70 95\%; Effective: the morphology of skin lesions subsided partially, the clinical symptoms improved partially, and the curative effect index ranged from 30-69\%; and Ineffective: the morphology and clinical symptoms of skin lesions hardly improved, or were even aggravated, and the curative effect index was less than $30 \%$. Total effective rate $=($ cure + markedly effective + effective) / all patients $\times 100 \%$. 
Table 1 Comparison of the baseline data between the two groups

\begin{tabular}{lcccc}
\hline Group & Age (years) & Gender (male/female) & Course of disease $($ years $)$ & BMl $\left(\mathrm{kg} / \mathrm{m}^{2}\right)$ \\
\hline Observation group $(\mathrm{n}=98)$ & $44.19 \pm 13.98$ & $58 / 40$ & $2.32 \pm 0.78$ & $23.51 \pm 2.79$ \\
Control group $(\mathrm{n}=97)$ & $43.31 \pm 14.40$ & $53 / 44$ & $2.29 \pm 0.73$ & $23.34 \pm 2.66$ \\
$t / \chi^{2}$ & 0.430 & 0.411 & 0.275 & 0.420 \\
$P$ & 0.668 & 0.522 & 0.784 & 0.675 \\
\hline
\end{tabular}

BMI, body mass index.

\section{Serum leukotrienes B4 (LTB4) and thymic stromal lymphocyte (TSLP) levels}

Two $\mathrm{mL}$ of fasting elbow vein peripheral blood was collected before and after treatment, and added to an EDTA-K2 anticoagulant (De sheng, China). A mikro220/220r centrifuge (Hettich, Germany) was used (parameters: 3,000 $\mathrm{rpm}$, time $=10 \mathrm{~min}$, inner diameter $=10 \mathrm{~cm}$ ). The supernatant was taken as the serum. The concentrations of serum LTB4 and TSLP were determined by BioTek synergy $\mathrm{H} 1$ automatic enzyme labeling instrument and enzyme-linked immunosorbent assay. The kit was purchased from Beijing Baiolabo Technology Co., Ltd. (China), and all operations were carried out according to the manufacturer's instructions.

\section{Adverse reaction}

Adverse reactions such as skin blister, scar, headache, palpitation, and fatigue were recorded.

\section{Factors influencing clinical efficacy}

Baseline data including gender, age, course of disease, digestive system symptoms, diet-induced, moistureinduced, fatigue aggravation, heavy aching limbs, reddish urine, thirst, skin lesion exudation, damp-heat tongue and pulse, and night aggravation were included. According to the clinical efficacy index, the efficacy of treatment in patients was divided into cure, markedly effective, effective, and ineffective levels. Taking the efficacy as the dependent variable, namely "total effective cases" and "ineffective cases", the independent influencing factors affecting the clinical efficacy were determined by single-factor and logistic regression analysis.

\section{Statistical analyses}

SPSS 22.0 statistical software (SPSS Inc., Chicago, IL, USA) was used for analysis. Measurement data that conformed to a normal distribution was expressed as $\bar{x} \pm \mathrm{S}$, and the t-test was adopted for comparison between groups. Count data was expressed as $\mathrm{n}(\%)$, and the $\chi^{2}$ test was used for comparison. The independent influencing factors affecting the clinical efficacy were determined by logistic regression analysis. A $\mathrm{P}$ value $<0.05$ for the difference was considered to be statistically significant.

\section{Results}

\section{General information}

A total of 195 patients with chronic eczema were included in this study. There were 98 patients in the observation group, including 58 males and 40 females, with an average age of $44.19 \pm 13.98$ years. There were 97 cases in the control group, including 53 males and 44 females, with an average age of $43.31 \pm 14.40$ years. The control group was treated with halometasone cream, while the observation group was treated with halometasone ointment combined with Simiao pill. There were no significant differences in the baseline data between the two groups in terms of age, sex, course of disease, and body mass index $(\mathrm{P}>0.05$, Table 1).

\section{Eczema lesion severity}

Before treatment, there was no significant difference in the eczema lesion severity scores between the two groups $(\mathrm{P}>0.05)$. After treatment, the EASI scores of both groups decreased significantly, and the EASI score of the observation group was significantly lower than that of the control group $(\mathrm{P}<0.05$, Table 2).

\section{Quality of life}

Before treatment, there were no significant differences in quality of life between the two groups $(\mathrm{P}>0.05)$. After treatment, the DLQI scores of patients in both groups 
Table 2 Comparison of the eczema severity scores between the two groups $\left(\bar{x}_{ \pm \mathrm{s}}\right)$

\begin{tabular}{lcccc}
\hline \multirow{2}{*}{ Group } & \multicolumn{2}{c}{ Eczema severity scores } & $t$ & After treatment \\
\cline { 2 - 4 } & Before treatment & $5.70 \pm 2.07$ & 18.436 & $<0.001$ \\
\hline Observation group $(\mathrm{n}=98)$ & $11.48 \pm 2.31$ & $9.11 \pm 1.86$ & 7.069 & $<0.001$ \\
Control group $(\mathrm{n}=97)$ & $11.15 \pm 2.25$ & 12.106 & \\
$t$ & 0.993 & $<0.001$ & \\
$\mathrm{P}$ & 0.322 & & \\
\hline
\end{tabular}

Table 3 Comparison of the quality of life between the two groups $\left(\bar{x}_{ \pm \mathrm{s}}\right)$

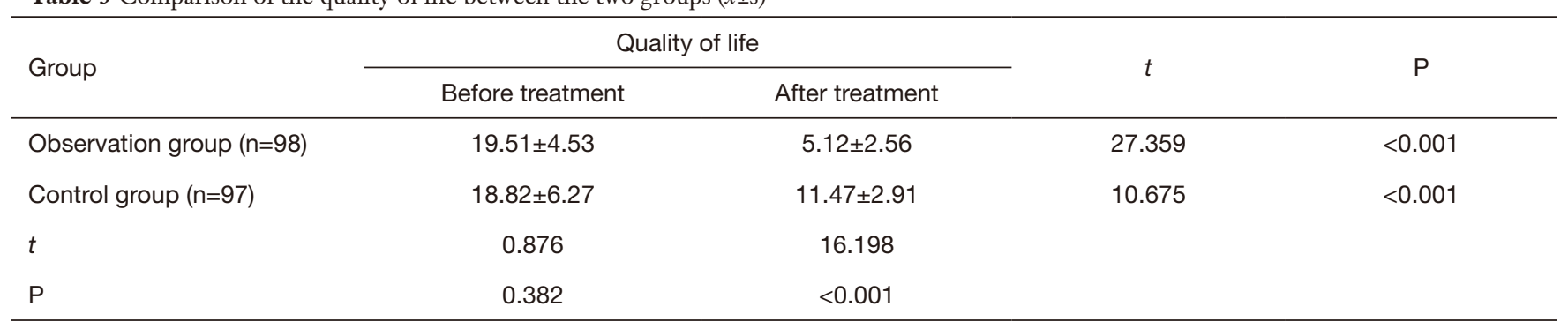

Table 4 Comparison of the clinical efficacy between the two groups

\begin{tabular}{lccccc}
\hline Group & Cure & Remarkable effect & Effective & Invalid & Total effective rate, $\mathrm{n}(\%)$ \\
\hline Observation group $(\mathrm{n}=98)$ & 59 & 24 & 4 & 11 & $87(88.8)$ \\
Control group $(\mathrm{n}=97)$ & 41 & 18 & 9 & 29 & $68(70.1)$ \\
$\chi^{2}$ & & & & 10.424 \\
$\mathrm{P}$ & & & & 0.001 \\
\hline
\end{tabular}

decreased significantly, and the DLQI scores of patients in the observation group were significantly lower than those in the control group $(\mathrm{P}<0.05$, Table 3).

\section{Comparison of clinical efficacy}

After treatment, the total clinical effective rate in the observation group was $88.8 \%$, which was significantly higher than that of the control group $(70.1 \%)\left(\chi^{2}=10.424\right.$, $\mathrm{P}=0.001$, Table 4, Figure 2).

\section{Comparison of serum LTB4 and TSLP levels}

Before treatment, there was no significant difference in serum LTB4 and TSLP levels between the two groups $(\mathrm{P}>0.05)$. After treatment, the concentrations of serum LTB4 and TSLP in the two groups decreased significantly, and the concentrations of serum LTB4 and TSLP in the observation group were significantly lower than those in the control group $(\mathrm{P}<0.05$, Table 5$)$.

\section{Comparison of adverse reaction}

During treatment, there were no adverse reactions such as skin blisters, scars, headache, palpitation, and fatigue in the two groups.

\section{Analysis of influencing factors of clinical efficacy}

The results of univariate analysis showed that there were statistically significant markers, such as digestive system symptoms, treatment plan, heavy aching limbs, diet induction, reddish urine, and damp-heat tongue and pulse $(\mathrm{P}<0.05$, Table 6). Logistic regression analysis was carried 

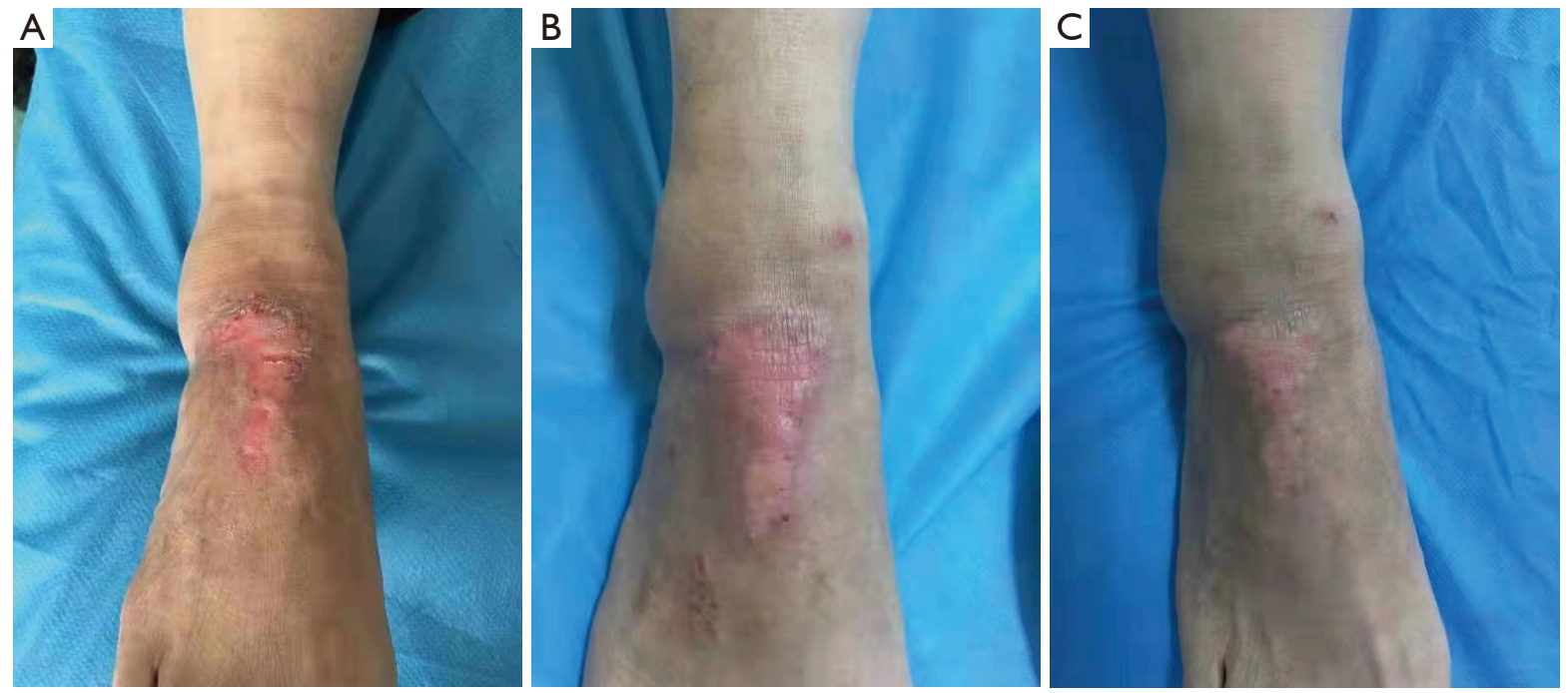

Figure 2 Representative photos of lesions before and after treatment of a patient from observation group. (A) Lesion on day 0; (B) lesion on day 7 ; (C) lesion on day 14.

Table 5 Comparison of serum LTB4 and TSLP levels between the two groups

\begin{tabular}{|c|c|c|c|c|c|c|c|c|}
\hline Group & \multicolumn{2}{|c|}{ LTB4, pg/mL } & $t$ & $\mathrm{P}$ & \multicolumn{2}{|c|}{ TSLP, pg/mL } & $t$ & $\mathrm{P}$ \\
\hline Observation group $(n=98)$ & $319.62 \pm 28.83$ & $146.92 \pm 20.25$ & 48.523 & $<0.001$ & $225.62 \pm 30.01$ & $118.30 \pm 24.78$ & 27.296 & $<0.001$ \\
\hline Control group ( $n=97)$ & $322.52 \pm 27.26$ & $210.87 \pm 26.19$ & 28.923 & $<0.001$ & $225.21 \pm 29.48$ & $161.23 \pm 32.84$ & 14.422 & $<0.001$ \\
\hline$t$ & 0.720 & 19.093 & & & 0.098 & 10.311 & & \\
\hline
\end{tabular}

LTB4, serum leukotrienes B4; TSLP, thymic stromal lymphocyte.

out on the indexes with statistically significant differences in the single-factor analysis (as the independent variable) and the effectiveness after treatment (as the dependent variable). The results showed that the treatment scheme [odds ratio $(\mathrm{OR})=2.401,95 \%$ confidence interval $(\mathrm{CI})$ : $1.585-3.638$ ], digestive system symptoms (OR $=2.018,95 \%$ CI: $1.071-3.800)$, heavy aching limbs $(\mathrm{OR}=2.770,95 \% \mathrm{CI}$ : 2.238-3.430), and damp-heat tongue and pulse (OR $=2.482$, 95\% CI: 1.373-4.486) were independent influencing factors affecting the curative effect of patients $(\mathrm{P}<0.05$, Table 7).

\section{Discussion}

Chronic eczema is a kind of inflammatory allergic disease, and its incidence is related to the interaction of genetic, immune, infection, gastrointestinal, and other factors. It has the characteristics of repeated attacks and a long treatment cycle, and has become one of the main skin diseases perplexing clinical medical staff and patients (12). Halometasone cream, as a common topical glucocorticoid, has good physiological anti-inflammatory functions, including inhibition of epidermal hyperplasia and immune control, but it has some dosage and location limitations (13). Traditional Chinese medicine has a long history and good curative effect in the treatment of eczema with little side effects. Simiao pill is composed of Amur Corktree Bark, Rhizoma Atractylodis, Semen Coicis, and Twotooth Achyranthes Root. It can dredge muscles and veins, clear heat, and dispel dampness. It has a certain effect on itching and pain and other skin diseases (14). Eczema is essentially an immune inflammatory disease, and LTB4 and TSLP are related to the body's immune or inflammatory response. However, there are few studies on the effect of Simiao pill combined with halometasone cream. Therefore, 
Table 6 Univariate analysis of clinical efficacy

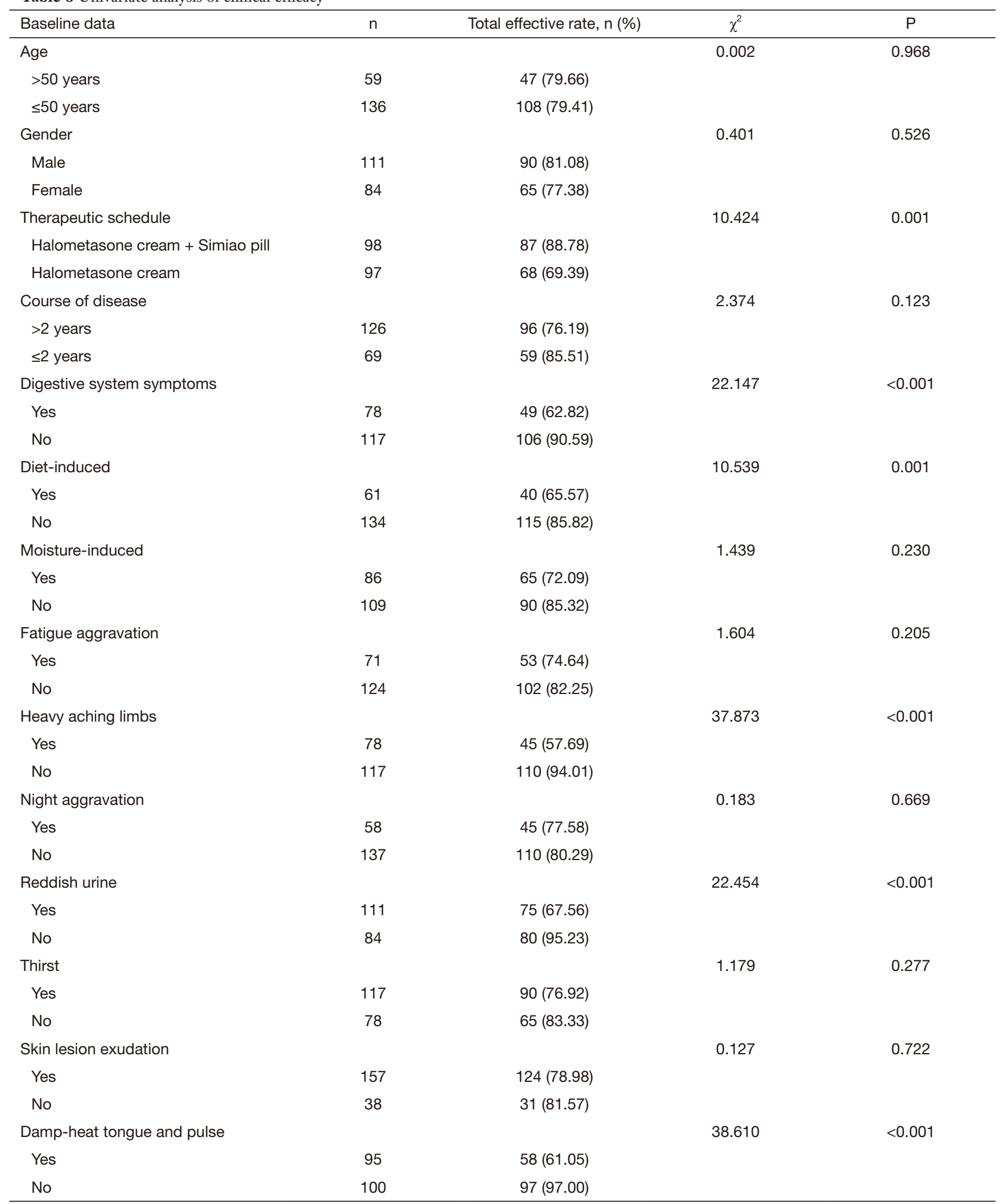


Table 7 Logistic regression analysis of clinical efficacy in patients

\begin{tabular}{lcccccc}
\hline Factor & $\beta$ & S.E. & Wald & $\mathrm{P}$ & OR & $95 \% \mathrm{Cl}$ \\
\hline Therapeutic schedule & 0.876 & 0.212 & 17.074 & $<0.001$ & 2.401 & $1.585-3.638$ \\
Digestive system symptoms & 0.702 & 0.323 & 4.724 & 0.030 & 2.018 & $1.071-3.800$ \\
Heavy aching limbs & 1.019 & 0.109 & 87.397 & $<0.001$ & 2.770 & $2.238-3.430$ \\
Damp-heat tongue and pulse & 0.909 & 0.302 & 9.060 & 0.003 & 2.482 & $1.373-4.486$ \\
Constant & -0.106 & 0.453 & 5.538 & 0.019 & - \\
\hline
\end{tabular}

this paper analyzed the effect of halometasone cream combined with Simiao pill on the treatment efficacy of eczema patients, the expression of serum LTB4 and TSLP, and the factors affecting the efficacy, in order to provide basis for clinical practice.

This study showed that after treatment, the EASI scores of the two groups decreased notably, while of the observation group was significantly lower than that of the control group $(\mathrm{P}<0.05)$. Also, the quality of life scores of patients in the observation group were markedly lower than those in the control group $(\mathrm{P}<0.05)$. At the same time, in terms of clinical efficacy, the total clinical effective rate of patients in the observation group was $88.8 \%$, which was significantly higher than that of the control group $(70.1 \%)$ $\left(\chi^{2}=10.424, \mathrm{P}=0.001\right)$, suggesting that Simiao pill combined with halometasone cream can effectively improve the degree of skin lesions and enhance the quality of life of patients. The serological indexes showed that after treatment, the concentrations of serum LTB4 and TSLP in the two groups decreased significantly, and the concentrations of serum LTB4 and TSLP in the observation group were markedly lower than those in the control group $(\mathrm{P}<0.05)$. This indicated that Simiao pill combined with halometasone cream may improve the total clinical effective rate by reducing the concentrations of serum LTB4 and TSLP. LTB4 is an inflammatory chemokine, which can mediate interleukin to promote the occurrence and development of eczema. At the same time, it can also combine with neutrophils to induce hypersensitivity and enhance the infiltration of inflammatory cells (such as lymphocytes) into the affected area of epidermis (15). TSLP is an important component of the body's immune function. It can regulate the expression of $\mathrm{T}$ cells and $\mathrm{B}$ cells in different microcirculation environments, mediate the involvement of $\mathrm{T}$ helper 2 (Th2) cells in allergic reactions, and aggravate chronic eczema (16).

Halometasone cream is a corticosteroid drug. It is a classic drug that regulates the immune and inflammatory response of the body, and can inhibit epidermal hyperplasia by reducing the inflammatory response and downregulating immune function, so as to improve eczema. In Simiao pill, Amur Corktree Bark exhibits antibacterial and anti-inflammatory properties, and plays a role in improving internal circulation, reducing vascular permeability, reducing the release of inflammatory factors, accelerating the growth of granulation tissue, and restoring the skin at the lesions (17). Pharmacological studies $(18,19)$ have shown that Rhizoma Atractylodis can optimize gastrointestinal function, regulate basic metabolic function, and enhance the ability of red blood cells to absorb and release oxygen. It can also cooperate with Amur Corktree Bark to inhibit the allergic immune response. Semen Coicis can down-regulate leukocyte chemotaxis, reduce the expression of cytokines (such as tumor necrosis factor and interleukin), and antagonize the body's inflammatory response. Moreover, Twotooth Achyranthes Root can enhance immune function, as well as provide calming and anti-inflammatory effects $(20,21)$. Therefore, Simiao pill combined with halometasone cream can improve clinical symptoms by down-regulating serum LTB4 and TSLP.

Logistic regression analysis showed that the treatment regimen $(\mathrm{OR}=2.401,95 \% \mathrm{CI}: 1.585-3.638)$, digestive system symptoms (OR $=2.018,95 \% \mathrm{CI}: 1.071-3.800)$, limb acid (OR $=2.770,95 \%$ CI: 2.238-3.430), and damp hot tongue and pulse $(\mathrm{OR}=2.482,95 \% \mathrm{CI}: 1.373-4.486)$ were independent influencing factors on the curative effect $(\mathrm{P}<0.05)$. Therefore, it is necessary to pay close attention to the above factors to improve the clinical curative effect.

In conclusion, Simiao pill combined with halometasone cream can effectively improve the symptoms of chronic eczema and the clinical efficacy, with high safety, which may be related to reducing the levels of serum LTB4 and TSLP. We found that the treatment scheme, digestive system symptoms, heavy aching limbs, and damp-heat tongue and 
pulse are the main factors affecting the clinical efficacy of patients. Thus, it is important to formulate the clinical intervention scheme according to the above factors to improve the quality of life of patients.

This study has some limitations that should be noted. Due to the small sample size of this study and the fact that patients were not prospectively randomized, selection bias cannot be ruled out. However, since there were no differences in preoperative characteristics between the patients in both groups, the results are unlikely to be misleading. It is expected that more in-depth research will be carried out in the future to verify the reliability of the research findings of this paper.

\section{Acknowledgments}

Funding: None.

\section{Footnote}

Reporting Checklist: The authors have completed the STROBE reporting checklist. Available at https://dx.doi. org/10.21037/apm-21-2685

Data Sharing Statement: Available at https://dx.doi. org/10.21037/apm-21-2685

Conflicts of Interest: All authors have completed the ICMJE uniform disclosure form (available at https://dx.doi. org/10.21037/apm-21-2685). The authors have no conflicts of interest to declare.

Ethical Statement: The authors are accountable for all aspects of the work in ensuring that questions related to the accuracy or integrity of any part of the work are appropriately investigated and resolved. The study was approved by the Ethics Committee of Wuxi 9th Affiliated Hospital of Soochow University (2020131) and was conducted in accordance with the Declaration of Helsinki (as revised in 2013). All participants provided informed consent.

Open Access Statement: This is an Open Access article distributed in accordance with the Creative Commons Attribution-NonCommercial-NoDerivs 4.0 International License (CC BY-NC-ND 4.0), which permits the noncommercial replication and distribution of the article with the strict proviso that no changes or edits are made and the original work is properly cited (including links to both the formal publication through the relevant DOI and the license). See: https://creativecommons.org/licenses/by-nc-nd/4.0/.

\section{References}

1. Raveendran R. Tips and Tricks for Controlling Eczema. Immunol Allergy Clin North Am 2019;39:521-33.

2. Barrett A, Hahn-Pedersen J, Kragh N, et al. PatientReported Outcome Measures in Atopic Dermatitis and Chronic Hand Eczema in Adults. Patient 2019;12:445-59.

3. Xu W, Li Y, Chen Z, et al. Wet-wrap therapy with halometasone cream for severe adult atopic dermatitis. Postgrad Med 2018;130:470-6.

4. Leins L, Orchard D. Eczema management in school-aged children. Aust Fam Physician 2017;46:896-9.

5. Li Y, Xu W, Li L. Efficacy and Safety of Halometasone Cream to Treat Chronic Generalized Eczema and the Effects of Halometasone Cream on Serum Cortisol Levels. Biomed Res Int 2017;2017:3265024.

6. Tian S, Bai M, Miao MS, et al. Analysis of eczema animal model based on clinical characteristics. Zhongguo Zhong Yao Za Zhi 2018;43:853-6.

7. Sun K, Wang X, Mei S. Study on the application effect of Simiao pills combined with setastine in the patients with scrotal eczema and influence for the expression of blood related indexes. Shaanxi Journal of Traditional Chinese Medicine 2019;40:1434-6.

8. Diepgen TL, Andersen KE, Chosidow O, et al. Guidelines for diagnosis, prevention and treatment of hand eczema. J Dtsch Dermatol Ges 2015;13:e1-22.

9. Chopra R, Vakharia PP, Sacotte R, et al. Severity strata for Eczema Area and Severity Index (EASI), modified EASI, Scoring Atopic Dermatitis (SCORAD), objective SCORAD, Atopic Dermatitis Severity Index and body surface area in adolescents and adults with atopic dermatitis. Br J Dermatol 2017;177:1316-21.

10. Li N, Teeple A, Muser E, et al. Use of the Dermatology Life Quality Index work/study domain to estimate overall work productivity loss among patients with psoriasis: an analysis based on real-world data. Clin Exp Dermatol 2020;45:572-5.

11. Patrizi A, Raone B, Ravaioli GM. Safety and Efficacy of Phototherapy in the Management of Eczema. Adv Exp Med Biol 2017;996:319-31.

12. Cazzaniga S, Apfelbacher C, Diepgen T, et al. Patterns of chronic hand eczema: a semantic map analysis of the CARPE registry data. Br J Dermatol 2018;178:229-37. 
13. Li L, Liang Y, Hong J, et al. The effectiveness of topical therapy combined with 308-nm excimer laser on vitiligo compared to excimer laser monotherapy in pediatric patients. Pediatr Dermatol 2019;36:e53-5.

14. Zhou L, Yang L, Huang Z. Professor Huang Zhengde's Clinical Experience in Using Simiao Pill in the Treatment of Various Diseases. Journal of Hunan University of Chinese Medicine 2019;39:341-4.

15. Liu M, Yokomizo T. The role of leukotrienes in allergic diseases. Allergol Int 2015;64:17-26.

16. Nitta T, Suzuki H. Thymic stromal cell subsets for T cell development. Cell Mol Life Sci 2016;73:1021-37.

17. Sun Y, Lenon GB, Yang AWH. Phellodendri Cortex: A Phytochemical, Pharmacological, and Pharmacokinetic Review. Evid Based Complement Alternat Med 2019;2019:7621929.

Cite this article as: Cao J, Liu H, Ding XJ. Effect of halometasone cream combined with Simiao pill on the therapeutic effect and expression of serum leukotriene B4 (LTB4) and thymic stromal lymphopoietin (TSLP) in patients with eczema, and the factors influencing its clinical efficacy. Ann Palliat Med 2021;10(11):11643-11652. doi: 10.21037/apm-212685
18. Chen LG, Jan YS, Tsai PW, et al. Anti-inflammatory and Antinociceptive Constituents of Atractylodes japonica Koidzumi. J Agric Food Chem 2016;64:2254-62.

19. Shim AR, Dong GZ, Lee HJ, et al. Atractylochromene Is a Repressor of Wnt/ $\beta$-Catenin Signaling in Colon Cancer Cells. Biomol Ther (Seoul) 2015;23:26-30.

20. Luo X, Li H, Jiang D, et al. Analysis of Fungi on Coix (Coix lacryma-jobi) Seed and the Effect of Its Aqueous Extract on the Growth of Aspergillus flavus. J Food Prot 2019;82:1775-82.

21. Zhang P, Meng X, Tang X, et al. The effect of a coix seed oil injection on cancer pain relief. Support Care Cancer 2019;27:461-5.

(English Language Editor: A. Kassem) 